\title{
Comparação do efeito de diferentes protocolos de polimento na rugosidade superficial de restaurações de resina composta
}

\author{
Comparison of the effect of different polishing protocols on surface rugosity of \\ composite resin restorations
}

\author{
Vivian Leite Martins ${ }^{1}$, Andrea Nóbrega Cavalcanti², Paula Mathias ${ }^{3 *}$ \\ ${ }^{1}$ Doutoranda em Processos Interativos dos Órgãos e Sistemas - ICS, UFBA. ${ }^{2}$ Professora Adjunta do Curso de \\ Odontologia, Escola Bahiana de Medicina e Saúde Pública e Universidade Federal da Bahia. ${ }^{3}$ Professora Adjunta do \\ Curso de Odontologia, Universidade Federal da Bahia.
}

\begin{abstract}
Resumo
Objetivo: o objetivo do presente estudo foi comparar a influência de diferentes protocolos de polimento sobre a rugosidade superficial de uma resina composta nanoparticulada. Metodologia: foram confeccionados 30 corpos de prova (1,5 mm espessura e $6 \mathrm{~mm}$ diâmetro), divididos em 3 grupos $(\mathrm{n}=10)$, de acordo com o tipo de polimento realizado: 1 . Grupo controle; 2 . Borrachas abrasivas em 3 granulações (grossa, média, fina); 3 Borracha abrasiva de granulação única. Todos os grupos receberam acabamento com lixa d'água, simulando uma ponta diamantada fina, sendo que o grupo controle não recebeu o polimento com borracha, após esse acabamento. Após o polimento dos grupos 2 e 3, a rugosidade superficial de cada corpo de prova, dos três grupos, foi avaliada em 3 medidas, através de rugosímetro, sendo calculada a média aritmética dos 3 valores picos e vales. A análise estatística inferencial foi feita por meio da ANOVA a 1-critério e teste de Tukey, para comparações múltiplas, com nível de significância de $5 \%$. Resultados: observaram-se maiores valores de rugosidade no grupo controle $(3,31 \mu \mathrm{m})$, seguido do polimento de 1 passo $(1,53 \mu \mathrm{m})$ e 3 passos $(0,48 \mu \mathrm{m})$. Conclusão: diante disso, observa-se alta rugosidade superficial no acabamento com pontas diamantadas, simulado pelo grupo controle, e sua redução após o polimento. Entretanto, apenas o sistema de 3 passos foi capaz de produzir uma lisura superficial clinicamente aceitável.
\end{abstract}

Palavras-chave: Polimento dentário. Resinas sintéticas. Nanopartículas.

\begin{abstract}
Aim: the aim of the present study was to compare the influence of different polishing protocols on the surface roughness of a nanoparticulate composite resin. Methodology: Thirty specimens $(1.5 \mathrm{~mm}$ thickness and $6 \mathrm{~mm}$ diameter) were made and divided into 3 groups $(n=10)$, according to the type of polishing performed: 1. Control group; 2 . 3 grit abrasive rubs (thick, medium, thin); 3 Single grain abrasive rubber. All groups were finished with sandpaper, simulating a fine diamond tip, and the control group did not receive rubber polishing after this finish. After groups 2 and 3 polishing, the surface roughness of each specimen of the three groups were evaluated in 3 measurements by means of roughness, and the arithmetic mean of the 3 peaks and valleys (Ra) values were calculated. Inferential statistical analysis was performed using one-way ANOVA and Tukey test for multiple comparisons, with a significance level of 5\%. Results: higher roughness values were observed in the control group $(3.31 \mu \mathrm{m})$, followed by 1-step $(1.53 \mu \mathrm{m})$ and 3-step $(0.48 \mu \mathrm{m})$ polishing. Conclusion: therefore, it is noticed a high surface roughness in the diamond-tipped finish, simulated by the control group, which was reduced after polishing. However, only the 3-step system was able to produce a clinically acceptable surface smoothness.

Keywords: Dental polishing. Dental resins. Surface properties. Nanoparticles.
\end{abstract}

\section{INTRODUÇÃO}

O sucesso clínico das resinas compostas está relacionado às demandas estéticas e de resistência. Correspondência de cores, estabilidade de cor, resistência mecânica e longevidade da restauração são importantes fatores que influenciam a falha ou o sucesso de uma restauração odontológica estética ${ }^{1-4}$.

Diversos fatores podem influenciar a qualidade das restaurações diretas em resina composta, dentre eles,

Correspondente/Corresponding: *Paula Mathias - End: Universidade de Odontologia da UFBA. Rua Araújo Pinho, 62, Canela, Salvador, Bahia. CEP: 40301-155 - Tel: (71) 99116-7275 - E-mail: pmathias@yahoo.com as técnicas de acabamento e polimento. A obtenção de lisura superficial é uma etapa operatória importante, uma vez que interfere significativamente na longevidade das restaurações, melhorando também sua aparência estética e sua resistência ${ }^{2,4-9}$.

O objetivo do acabamento é obter a forma e a adaptação anatômica desejadas, contornando a restauração e gerando o perfil de emergência, ajuste marginal, forma e oclusão adequados. Além disso, a etapa do acabamento é responsável pela reprodução das anatomias primária, secundária e terciária, que reproduzem a textura de superfície natural nos dentes restaurados. Tais ajustes são normalmente realizados com instrumentos de corte 
rotatórios, como pontas diamantadas de granulações fina e/ou extrafinas ou brocas multilaminadas, que modificam as características da superfície da restauração, alterando a topografia superficial e causando um aumento na rugosidade da resina ${ }^{7-9}$

Após o acabamento e a reprodução dos detalhes anatômicos, é indicado o uso de discos de lixa, borrachas abrasivas, escovas e pastas de diamante, no intuito de polir a restauração e obter uma maior lisura superficial 4,7 . No entanto, são poucas as evidências científicas que indicam quais técnicas e materiais de acabamento e polimento seriam os mais eficazes para as resinas compostas, especialmente as resinas nanoparticuladas ${ }^{4,5,6,8}$. 0 tipo de resina usada também influencia a lisura das restaurações, considerando fatores como composição da matriz e conteúdo de carga das resinas, sendo as nanoparticuladas as que apresentam maior lisura superficial, uma vez que apresentam alto grau de polimento inicial e manutenção desse polimento, além de excelentes propriedades mecânicas ${ }^{1,3,4,10,11}$.

Dessa forma, a rugosidade da superfície pode ser determinada, tanto pelas características intrínsecas do compósito quanto pelos procedimentos de acabamento e polimento usados. Portanto, uma restauração bem sucedida requer cuidados com a escolha do material restaurador, associado a um bom sistema de acabamento e polimento ${ }^{4,10,11}$.

Anteriormente, o uso sequencial de discos e borrachas abrasivas de granulações mais grossas para granulações mais finas, em várias etapas, era recomendado para obter restaurações de resina composta altamente polidas. Entretanto, a ênfase atual é baseada em sistemas de etapas reduzidas que combinam alguns dos procedimentos de acabamento e polimento em uma ou duas etapas $^{12}$. Apesar de a simplificação ser uma tendência desejável, é preciso verificar se ela resulta na obtenção de uma lisura superficial semelhante à obtida com um polimento multipassos, onde três diferentes granulações de borracha são utilizadas.

Diante disso, o objetivo do presente trabalho foi comparar a influência de diferentes protocolos de polimento sobre a rugosidade superficial de uma resina composta nanoparticulada, submetida a procedimentos de polimento, com borrachas abrasivas de três diferentes granulações; e com borracha abrasiva de granulação única. A hipótese nula testada é que não haverá diferença na rugosidade superficial da resina nanoparticulada entre os diferentes protocolos de polimento.

\section{METODOLOGIA}

\section{Confecção dos corpos de prova}

Trinta corpos de prova em resina composta nanoparticulada (Vittra APS, cor E bleach, FGM, Joinville-SC) foram confeccionados, usando-se uma matriz de aço inox, com
1,5 $\mathrm{mm}$ de espessura e $6 \mathrm{~mm}$ de diâmetro. Nesse molde, a resina composta foi acomodada individualmente em um incremento único, sendo colocada sobre o conjunto uma tira de poliéster e, sobre ela, um peso de $500 \mathrm{~g}$, mantido por 30 segundos, para permitir melhor acomodação do material. Após esse período, a superfície de topo foi fotoativada com luz LED (Valo Cordless $1400 \mathrm{~mW} / \mathrm{cm}^{2}$, Ultradent, South Jordan, EUA) por 20 segundos, com a ponta dispensadora de luz mantida em contato direto com a tira. Após confeccionados, os corpos de prova foram armazenados por 24 horas, a $37^{\circ} \mathrm{C}$ em umidade relativa, livres do contato com a luz.

Figura 1 - Corpos de prova de resina composta nanoparticulada inseridos em resina de poliestireno.

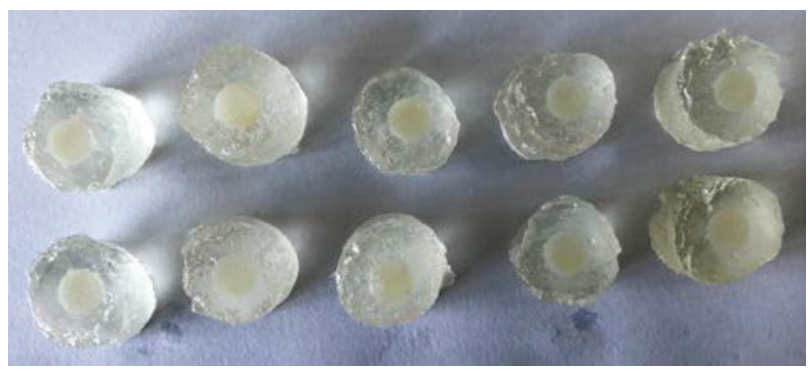

Fonte: As autoras.

\section{Protocolos de acabamento e polimento}

Após o período de armazenamento, os corpos de prova foram incluídos em resina de poliestireno e receberam acabamento inicial na superfície superior, com lixas d'água de granulação 120 (Bosch Brasil, São Paulo - SP), para simulação do acabamento com brocas multilaminadas ${ }^{1}$.

Posteriormente, foram divididos de forma aleatória, em três grupos $(n=10)$, de acordo com o protocolo de polimento utilizado, seguindo as recomendações do fabricante:

- Ausência de polimento: os corpos de prova não receberam nenhum tipo de polimento.

- Borrachas abrasivas em três granulações (grossa, média, fina): em cada corpo de prova foi realizada a sequência de polimento com as borrachas abrasivas, em formato de chama, do sistema Ultra Gloss (American Burrs), da granulação mais grossa (cinza) para a mais fina (azul), utilizando-se baixa rotação, durante 30 segundos cada. Após cada etapa, o corpo de prova foi lavado com jato de ar/água durante 10 segundos e seco com jato de ar por 10 segundos.

- Borrachas abrasivas em uma granulação: cada corpo de prova recebeu o polimento com borracha abrasiva do sistema Dura-Gloss One-Step (American Burrs), em formato de chama, durante 30 segundos, seguido da lavagem com jato de ar/água durante 10 segundos e secagem com jato de ar por 10 segundos.

Todos os procedimentos foram realizados pelo mesmo pesquisador, para redução de variabilidade na 
técnica. Além disso, todos os materiais utilizados para o polimento foram trocados após o polimento de cinco corpos de prova.

Figura 2 - Sistema de polimento Dura Gloss de passo único (A); Sistema de polimento Ultra Gloss de 3 passos (B)
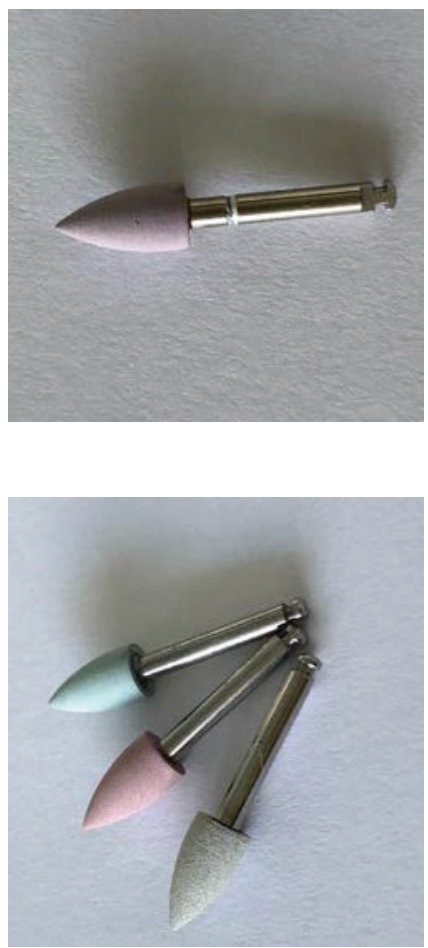

Fonte: As autoras.

\section{Avaliação da rugosidade superficial}

A rugosidade da superfície de topo de cada corpo de prova foi individualmente avaliada, usando o rugosímetro (Surftest 3000, Mitutoyo Sul Americana, São Paulo, SP, Brasil). O valor considerado para cada leitura foi a média aritmética entre picos e vales $(\mathrm{Ra})$, registrada num trecho de $1,25 \mathrm{~mm}$, numa velocidade de $0,1 \mathrm{~mm} / \mathrm{s}$. Foram realizadas três leituras por superfície, em posições diferentes, para maior abrangência da área testada. $O$ valor de rugosidade superficial foi obtido através da média aritmética das três leituras

\section{Análise Estatística}

Inicialmente, foi realizada a análise exploratória dos dados de rugosidade para verificação da presença de normalidade (Shapiro-Wilk; $p>0,05$ ) e demais parâmetros da ANOVA. A análise estatística inferencial foi realizada pela ANOVA 1-critério. Para comparações múltiplas entre as médias, foi utilizado o teste Tukey. As análises foram realizadas no programa estatístico SAS, versão 9.1, com nível de significância de 5\%.

\section{RESULTADOS}

A Tabela 1 apresenta a média e o desvio-padrão obtidos pela variável rugosidade.

De acordo com a análise estatística, verificou-se diferença estatisticamente significativa entre os grupos experimentais $(p<0,0001)$ entre $s i$, indicando maiores valores de rugosidade no grupo controle, seguido do sistema de polimento de um passo (Dura Gloss) e do sistema de polimento de três passos (Ultra Gloss).

Tabela 1 - Média (desvio padrão) dos valores de delta.

\begin{tabular}{lll}
\hline Grupos/Polimento & $\begin{array}{l}\text { Média (desvio-padrão) do Ra Tukey } \\
\text { em } \mu \mathrm{m}\end{array}$ \\
\hline Controle & $3,31(0,68)$ & $\mathrm{A}$ \\
1 passo (Dura Gloss) & $1,53(0,53)$ & $\mathrm{B}$ \\
3 passos (Ultra Gloss) & $0,48(0,09)$ & $\mathrm{C}$ \\
\hline
\end{tabular}

Fonte: Dados da pesquisa.

Legenda: Letras distintas representam significância estatística entre os grupos (1-way ANOVA/Tukey, alfa=5\%).

\section{DISCUSSÃO}

Dentre as técnicas de polimento, as borrachas abrasivas são comumente utilizadas e podem ser aplicadas em diferentes protocolos e impregnadas com diferentes abrasivos. Segundo o fabricante, as borrachas de segunda geração são compostas por duas camadas de silicone impregnadas com carbeto de silício e são dispostas em três granulometrias: grossa, média e fina, que devem ser usadas em sequência, com movimentos intermitentes, para evitar superaquecimento, sempre lavando entre as etapas. As borrachas de terceira geração são polidores impregnados com nanopartículas de diamantes naturais que, segundo o fabricante, promovem uma distribuição mais homogênea das partículas na superfície, menor aquecimento do material restaurador e polimento em uma única etapa.

O presente estudo avaliou, por meio da rugosidade superficial, o grau do polimento de dois sistemas de borrachas abrasivas, comparando o sistema de um passo, com o de três passos e tendo como controle a ausência de polimento. A hipótese nula testada no presente estudo foi negada, uma vez que o sistema de polidores de três passos demonstrou superioridade sobre o sistema de passo único e também sobre o grupo controle.

Foram observados ainda maiores valores de rugosidade superficial no grupo controle, seguido do polimento com borrachas abrasivas do sistema de um passo (Dura Gloss) e do sistema de três passos (Ultra Gloss). Esse resultado pode ser justificado pelo fato de as partículas de diamante impregnadas na borracha do sistema de um passo serem mais duras, o que pode ser prejudicial às partículas de vidro presentes na matriz inorgânica da resina, formando uma superfície mais porosa e levemente riscada, consequentemente mais rugosa ${ }^{13-15}$. 
Resultados diferentes foram obtidos no estudo de Carneiro e colaboradores ${ }^{6}$, o qual avaliou diferentes sistemas de acabamento e polimento sobre três tipos de resina composta e concluiu que o número de etapas de aplicação não tem efeito no desempenho dos sistemas de acabamento e polimento. Além disso, segundo os autores, polidores de passo reduzido usados depois de um acabamento prévio podem ser preferíveis aos sistemas de vários passos, quando usados sobre resina composta nanohíbrida. Dessa forma, é possível supor que, no presente estudo, o sistema de três passos obteve melhores resultados de rugosidade, quando comparado ao de passo único, pois este último depende de um acabamento prévio mais refinado, o que não foi realizado na presente metodologia, sendo realizada apenas simulação do uso de pontas diamantadas.

A rugosidade de superfície de uma restauração em resina composta está diretamente relacionada com a flexibilidade do material em que os abrasivos estão impregnados e da dureza e tamanho das partículas desses abrasivos $^{15}$. Segundo alguns autores ${ }^{12,16}$, o limiar de rugosidade superficial para retenção de placa bacteriana é de 0,2 $\mu \mathrm{m}$. No entanto, outros autores não encontraram diferenças significativas na retenção de placa em superfícies com valores de Ra que variaram de 0,7 a 1,4 $\mu \mathrm{m}$ e descobriram que as restaurações parecem opticamente lisas quando o Ra é menor que $1 \mu \mathrm{m}^{7,10}$. Assim, a resina composta usada neste estudo produziu valores aceitáveis de $\mathrm{Ra}$, do ponto de vista clínico, apenas para o sistema de polimento Ultra Gloss $(0,48 \mu \mathrm{m})$, sendo possível dizer que o sistema Dura Gloss, com média de Ra 1,53 $\mu$ m apresenta rugosidade "visivelmente suave"12. Em relação ao grupo controle, pode-se observar que a simulação da utilização apenas de pontas diamantadas finas para acabamento e polimento da resina acarretou a formação de uma superfície muito rugosa $(3,31 \mu \mathrm{m})$, o que pode comprometer a qualidade estética da restauração, além de facilitar o acúmulo de biofilme.

Outros problemas relacionados ao acabamento e polimento inadequados, como as alterações na cor e na rugosidade superficial das resinas compostas, estão também relacionados à qualidade de fotopolimerização e à forma como a etapa de acabamento e polimento é realizada, além da composição do material, onde a estrutura da matriz resinosa e as características da partícula de carga têm um impacto direto na lisura superficial desses materiais ${ }^{2,12}$. Dessa forma, resinas com cargas de maior tamanho mostram maior rugosidade da superfície, quando comparadas àquelas com cargas de menor tamanho. Esses compósitos contendo partículas pequenas, como os nanoparticulados e nanohíbridos, são mais resistentes ao desgaste superficial por apresentarem superfície mais homogênea, ou seja, menor protrusão das partículas na superfície, resultando em menor fricção e abrasão do compósito ${ }^{1,4}$ 6, 10,11.

Apesar dos resultados obtidos mostrarem uma superioridade do sistema multipassos para polimento da resina composta nanoparticulada, mais estudos, envolvendo diferentes tipos de resina composta e outros sistemas de polimento são necessários. No entanto, é importante ressaltar a importância de uma boa técnica de acabamento e polimento para a longevidade e maior qualidade das restaurações diretas em resina composta, assim como a sua manutenção e acompanhamento.

\section{CONCLUSÃO}

Considerando as limitações do presente estudo, é possível concluir que o acabamento apenas com pontas diamantadas gera altos valores de rugosidade superficial. Entretanto, a associação desse acabamento a outros sistemas de polimento é capaz de produzir uma maior lisura superficial.

Em relação aos sistemas de polimento, é possível concluir que o sistema de três passos apresentou maior lisura superficial devido à ausência de um acabamento mais refinado, que comprometeu a obtenção de uma lisura adequada no sistema de passo único.

\section{AGRADECIMENTOS}

Os autores agradecem ao Curso de Odontologia e ao Instituto de Ciências da Saúde da Universidade Federal da Bahia; à American Burrs, pela doação dos materiais; e aos responsáveis pelo Laboratório de Biomateriais da Faculdade de Medicina e Saúde Pública da Bahia, pela parceria e disponibilidade.

\section{REFERÊNCIAS}

1. ANTONSON, S. et al. Comparison of different finishing/polishing systems on surface roughness and gloss of resin composites. J. Dent., [s.I], n.39, pe9-e17, 2011.

2. BARAKAH, H.M.; TAHER, N.M. Effect of polishing systems on stain susceptibility and surface roughness of nanocomposite resin material. J. prosthet. dent., St. Louis, v. 112, n. 3, p.625-631, Sept. 2014. DOI: 10.1016/j.prosdent.2013.12.007.

3. COSTA, J. et al. The effect of different polishing systems on surface roughness and gloss of various resin composites. J. esthet. restor. dent., Hamilton, n. 19, p. 214-226, 2007.

4. MODA, M. D. et al. Comparison of different polishing methods on the surface roughness of microhybrid, microfill, and nanofill composite resins. J. investig. clin. dent., Richimond, e122-187, 2017.

5. BARBOSA, S.L. et al. Effect of different finishing and polishing techniques on the surface roughness of microfilled, hybrid and packable composite resins. Braz. dent. j., Ribeirão Preto, v. 16, n. 1, p. 39-44, 2005.

6. CARNEIRO, P. M. A. et al. Influence of finishing and polishing techniques and abrasion on transmittance and roughness of composite resins. Oper. Dent., Seattle, v. 41, n. 6 p. 634-641, 2016.

7. DUTRA, D.A.M. et al. Does finishing and polishing o restorative materials affect bacterial adhesion and biofilm formation? A systematic review. Oper. Dent., Seattle, v. 43, n. 1, p. 37-52, 2018.

8. JANG, J.H. et al. Clinical effectiveness of different polishing systems and self-etch adhesives in class $v$ composite resin restorations: two-year randomized controlled clinical trial. Oper. Dent., Seattle, v. 42, n. 1, p. 19-29, 2017. 
9. RUSCHEL, V.C. et al. Effect of surface sealants and polishing time on composite surface roughness and microhardness. Oper. Dent., Seattle, v. 43, n. 4, p. 408-415, July/Aug. 2018. DOI: 10.2341/17-048-L.

10. PALA, K. et al. Evaluation of the surface hardness, roughness, gloss and color of composites after different finishing/polishing treatments and thermocycling using a multitechnique approach. Dent. Mater. J., Tokyo, v. 35, n. 2, p. 278-289, 2016.

11. KAIZER, M.R. et al. Do nanofill or submicron composites show improved smoothness and gloss? A systematic review of in vitro studies. Dent. Mater., Washington, n. 30, p. e41-78, 2014.

12. KEMALOGLU, H.; KARACOLAK, G.; TURKUN, L. S. Can reduced-step polishers be as effective as multiple-step polishers in enhancing surface smoothness? J. esthet. restor. dent., Hamilton, v. 29, n.1, p. 31-40, 2016.
13. LU, H.; ROEDER, L.B.; POWERS, J.M. Effect of polishing systems on the surface roughness of microhybrid composites. J. esthet. restor. dent., Hamilton, n. 15, p.297-303, 2003.

14. JUNG, M.; EICHELBERGER, K.; KLIMEK, J. Surface geometry of four nanofiller and one hybrid composite after one-step and multiple-step polishing. Oper. Dent., Seattle, v. 32, n. 4, p. 347-355, 2007.

15. AVSAR, A.; YUZBASIOGLU, E.; SARAC, D. The effect of finishing and polishing techniques on the surface roughness and the color of nanocomposite resin restorative materials. Adv. Clin. Exp. Med., Wroclaw, v. 24, n.5, p. 881-990, 2015.

16. ENDO, T. et al. Surface texture and roughness of polished nanofill and nanohybrid resin composites. Dent. mater. j., Tokyo, v. 29, n. 2, p. 213-223, 2010.

Submetido em: 07/11/2019

Aceito em: 29/11/2019 